\title{
The influencing mechanism of reward on executive function in heroin addicts
}

\author{
Yang Ling, Cao Hua*, He Yuan, Yuan XU and Qiong Ying \\ School of Psychology, Northwest Normal University, Lanzhou 730070, China
}

\begin{abstract}
Research has identified several features of heroin-related reward dysfunction, including the enhanced attentional bias for heroin-related cues, increased drug craving, preference for immediate over delayed rewards of larger value and decreased sensitivity to delayed consequences. It has also been found that heroin addicts exhibit executive dysfunction. Reward dysregulation and executive function deficits have been hypothesized to play an important role in the maintenance of drug taking and abstinence. However, it is not clear yet how reward influences executive function. We want to investigate the effect of heroin-related cues and monetary reward on executive function in heroin addicts after different periods of abstinence. The results will not only contribute to the development of the addiction theories, but also help to identify the risk factors and the proper objectives in different abstinent periods.
\end{abstract}

Executive function deficits and reward dysregulation, are well documented in drug abusers [1]. The changes of brain reward system caused by chronic substance use are considered central to the development and maintenance of addiction [2,3]. Addicts show a preference for substance-related incentives and a decreased interest in non-substance-use behaviors, which is a disorder and, undoubtedly, one of the most important causes of an individual's dependence on addictive substances [4]. Most recent studies have shown that the reward function of heroin addicts is abnormal. Reward incentives for drug addicts can be divided into drug rewards and non-drug rewards. Non-drug rewards are natural rewards which are generally divided into primary rewards (eg. food, water, etc.) and secondary rewards (eg. money, power, etc.). On monetary rewards, studies had shown that the heroin addicts' delay discount rates of monetary rewards were higher than normal people [5] and in EEG studies found that heroin addicts had abnormal EEG changes in the task on money reward processing. On drug rewards, the studies showed that the delay discount rate of heroin addicts was significantly higher than monetary rewards, indicating that heroin addicts have an "immediate yield first" high-risk decision-making model and a reduced sensitivity on long-term gains, and a high sensitivity on drug rewards [7]. Therefore, heroin addicts have unusual rewards processing, whether on money or drug rewards.

Executive functions, also known as executive control or cognitive control function, is a human advanced cognitive function, which can regulate a variety of cognitive processes, achieving top-down regulation of behavior [8,9]. defined executive function as coordinating various cognitive processes in the completion of complex cognitive tasks, thereby ensuring that the cognitive system exercises a general control over a particular goal in a flexible and optimized manner. The essence is to control and regulate other cognitive processes, and the fundamental purpose is to produce coordinated, orderly, and purposeful behavior. Researchers generally agree that executive functions involve three basic sub-functions: Shifting between tasks or mental sets; updating and monitoring of working memory representations; and inhibition of prepotent responses [8]. The executive dysfunction of heroin addicts has been confirmed by numerous studies. It is thought to be associated with some brain damage in the prefrontal cortex, thereby reducing the ability of drug users to regulate their own behavior [10], producing continuous medication, relapse after withdrawal and some criminal activities. However, the current research mainly focused on the inhibition. At the behavioral level, the study found that the response inhibition ability of heroin addicts was impaired, for example, the response time in the Stroop task was longer and the error rates were higher [11]. At the electroencephalogram level, the study used the Stroop task and found that there was a disappearance of the $\mathrm{N} 2$ effect of heroin addicts at the conflict monitoring processing stage and a disappearance of SP effect during the process of conflict resolution. The researchers believe that the heroin addicts may have the early conflict monitoring disorder and the late response conflict to solve the abnormal processing. This may be due to long-term abuse of heroin, which causes damage to brain function. Brain imaging studies have shown that cognitive activation-related brain structure (such as prefrontal cortex, PFC) activation is attenuated in heroin addicts during response suppression tasks, whether in withdrawal period [12] or nonwithdrawal period [13]. In the heroin addicted population, there are relatively few studies on other sub-functions relating to suppression of executive function, but the studies also found that heroin addicts had flaws while involving in cognitive flexibility, attention, conversion, decision making, working memory, etc $[6,11,14,15]$.

The dual competition model [16] argues that the interaction between emotion and motivation, and executive control determines the outcome of behavior. The emotion and motivation will affect the perceived and enforced competition. There are two ways in

Correspondence to: Cao Hua, School of Psychology, Northwest Normal University, Lanzhou 730070, China, Email: caohua360083915@163.com

Key words: executive function; reward dysregulation; heroin-related cues; money incentive; heroin addicts

Received: January 24, 2018; Accepted: February 15, 2018; Published: February 19,2018 
which motivation has influence on the executive function: First, the motivation of ascension leads to the enhancement of the executive function through the influence of the orientation and the reorientation of the attention. For example, the study found that rewards can increase the individual's conflict adaptation [17]. Second, in order to maximize the rewards, motivation can be redistributed to processing resources that perform functions. For instance, the study found that rewards led to a decrease in the inhibition of individuals in the stop-signal task [18] and working memory tasks switch cost increases [17]. Some addiction theories also emphasize the interaction between reward disorders and executive dysfunction on the basis of the development and maintenance of drug addiction behaviors [19].

Heroin addicts have some abnormalities in reward features [20] and executive function [21]. Reward incentives will drive behavior, while the individual's executive function will be adjusted by weighing the individual's behavior. The two interact with each other to determine the behavior [16]. Although some addictive theories have proposed an integrated view that drug-related clues and executive control and their interactions play an important role in the continued drug use and relapse among drug addicts $[10,19]$ in other studies of substance addiction, drug-related clues have been shown to decrease individual response inhibition [22]. The regulation of the executive function of cocaine-addicted individuals of monetary rewards are abnormal [23]. However, the impact of drug-related clues on the executive function of people with substance addiction is also dependent on the substance [24] or the severity of the drug used [25].

Few studies have investigated the impact of reward executive function in heroin addicts whiles the findings of related studies were not consistent, and limited to the behavioral level, lacking the indepth exploration of cognitive neurosurgery. Monetary rewards and drug-related clues tend to be an important aspect of rewards. Now, no researcher has investigated the impact of rewards on executive functions based on drug-related clues and money rewards expectations, and the mechanism of such effects in different addictive drugs, and no one has investigated the mechanism by which rewards of heroin addicts affect executive function. Also, the length of withdrawal time is an important factor for the addicts to perform functional impairment and functional recovery after withdrawal [26] but this is seldom included in studies as an important variable by researchers. Based on this, we are very interested in investigating the mechanism of executive function impact of rewards for heroin addicts under drug-induced clues and monetary reward as well as the impact of such occurrences on withdrawal time.

\section{References}

1. Morie KP, De SP, Garavan H, Foxe JJ (2014) Executive dysfunction and reward dysregulation: a high-density electrical mapping study in cocaine abusers. Neuropharmacology 85(10): 397-407. [Crossref]

2. Koob GF, Volkow ND (2010) Neurocircuitry of addiction. Neuropsychopharmacology 35: 217-238. [Crossref]

3. Noël X, Brevers D, Bechara A (2013) A neurocognitive approach to understanding the neurobiology of addiction. Curr Opin Neurobiol 23: 632-638. [Crossref]

4. Ling Y, Bobo S, Jianxun Z, Bin L, Xiaoyun W, Xin Z (2015) Dysfunction of Monetary Reward Processing and Recoverability in Drug Addicts. Advances in Psychological Science 23(9): 1617-1626.

5. Kirby KN, Petry NM, Bickel WK (1999) Heroin addicts have higher discount rates for delayed rewards than non-drug-using controls. $J$ Exp Psychol Gen 128: 78-87. [Crossref]
6. Al-Zahrani, Mohamed A, Elsayed, Yasser A (2009) The impacts of substance abuse and dependence onneuropsychological functions in a sample of patients from Saudi Arabia. Behavioral \& Brain Functions 5(1): 322-326. [Crossref]

7. Madden, Gregory J, Petry, Nancy M, Badger, et al. (1997) Impulsive and self-contro choices in opioid-dependent patients and non-drug-using control patients: Drug and monetary rewards. Experimental \& Clinical Psychopharmacology 5(3): 256-262. [Crossref]

8. Jarmolowicz, David P, Mueller, E Terry, Koffarnus, Mikhail N, et al. (2013) Executive dysfunction in addiction. The Wiley-Blackwell Handbook of Addiction Psychopharmacology 27-61.

9. Baddeley Alan (1996) Exploring the central executive. The Quarterly Journal of Experimental Psychology: Section A 49(1) 5-28.

10. Goldstein RZ, Volkow ND (2011) Dysfunction of the prefrontal cortex in addiction: neuroimaging findings and clinical implications. Nat Rev Neurosci 12(11): 652-669. [Crossref]

11. Suzan, Hekmat, Zahra, Mehrjerdi A (2011) Cognitive Flexibility, Attention and Speed of MentalProcessing in Opioid and Methamphetamine Addicts in Comparison with Non-Addicts. Basic \& Clinical Neuroscience 2 (2): 12-19.

12. Fu, Li-ping, Bi, Guo-hua, Zou, Zhi-tong, et al. (2008) Impaired response inhibition function in abstinent heroin dependents: an fMRI study. Neuroscience letters 438(3): 322-326. [Crossref]

13. Lee, Tatia MC, Wen-Hua, Zhou, Xiao-Jing, et al. (2005) Neural activity associated with cognitive regulation in heroin users: A fMRI study. Neuroscience Letters 382(3): 211-216. [Crossref]

14. Fishbein, Diana H, Krupitsky, Evgeny A, Flannery B, Barbara A, et al. (2007) Neurocognitive characterizations of Russian heroin addicts without a significan history of other drug use. Drug \& Alcohol Dependence 90(1): 25-38. [Crossref]

15. Zahra, Mehrjerdi A, Sara, Bakhshi, Siavash, et al. (2011) The Impact of Hydrochloride Heroin on Mental Flexibility, Abstract Reasoning, Impulsivity and Attention. Basic \& Clinical Neuroscience 2 (3): 27-32.

16. Pessoa L (2009) How do emotion and motivation direct executive control? Trends Cogn Sci 13(4): 160-166. [Crossref]

17. Braem S, Tom V, Chantal R (2012) Reward modulates adaptations to conflict Cognition 125(2): 324-332. [Crossref]

18. Padmala S, Pessoa L (2010) Interactions between cognition and motivation during response inhibition. Neuropsychologi 48(2): 558-565. [Crossref]

19. Field M, Cox WM (2008) Attentional bias in addictive behaviors: a review of its development, causes, and consequences. Drug Alcohol Depend 97: 1-20. [Crossref]

20. Franken IH, Kroon LY, Wiers RW, Jansen A (2000) Selective cognitive processing of drug cues in heroin dependence. Journal of Psychopharmacology 14(4): 395-400. [Crossref]

21. Yang L, Zhang J, Zhao X (2015) Implicit processing of heroin and emotional cues in abstinent heroin users: early and late event-related potential effects. Am J Drug Alcohol Abuse 41(3): 237-245. [Crossref]

22. Fanny K, Aurélie V, Etienne Q (2013) Response inhibition toward alcohol-related cues using an alcohol go/no-go task in problem and non-problem drinkers. Addictive Behaviors 38(10): 2520-2528. [Crossref]

23. Hester R, Bell RP, Foxe JJ, Garavan H (2013) The influence of monetary punishment on cognitive control in abstinent cocaine-users. Drug Alcohol Depend 133(1): 86-93.

24. Luijten M, Little M, Franken IH (2011) Deficits in Inhibitory Control in Smokers During a Go/NoGo Task: An Investigation Using Event-Related Brain Potentials. Plos One 6(4): e18898. [Crossref]

25. Géraldine P, Charles K, Xavier N, Paul V, Salvatore C (2012) Alcohol-Related Context Modulates Performance of Social Drinkers in a Visual Go/No-Go Task: A Preliminary Assessment of Event-Related Potentials. Plos One 7(5): e37466. [Crossref]

26. Lou M, Wang E, Shen Y, Wang J (2012) Cue-Elicited Craving in Heroin Addicts at Different Abstinent Time: An fMRI Pilot Study. Substance Use \& Misuse 47(6): 631639. [Crossref]

Copyright: (C2018 Ling Y. This is an open-access article distributed under the terms of the Creative Commons Attribution License, which permits unrestricted use, distribution, and reproduction in any medium, provided the original author and source are credited. 